\title{
Energy Absorption as a Predictor of Leg Impedance in Highly Trained Females
}

By: Anthony S. Kulas, ${ }^{1,2}$ Randy J. Schmitz, ${ }^{2}$ Sandra J. Shultz, ${ }^{2}$ Mary Allen Watson, ${ }^{2,3}$ and David H. Perrin ${ }^{2}$

Kulas, A.S., Schmitz, R.J., Shultz, S.J., Watson, M.A., Perrin, D.H. (2006). Energy absorption as a predictor of leg impedance in highly trained females. Journal of Applied Biomechanics, 22:177-185.

Made available courtesy of Human Kinetics: http://www.humankinetics.com/

***Note: Figures may be missing from this format of the document

\begin{abstract}
:
Although leg spring stiffness represents active muscular recruitment of the lower extremity during dynamic tasks such as hopping and running, the joint-specific characteristics comprising the damping portion of this measure, leg impedance, are uncertain. The purpose of this investigation was to assess the relationship between leg impedance and energy absorption at the ankle, knee, and hip during early (impact) and late (stabilization) phases of landing. Twenty highly trained female dancers $($ age $=20.3 \pm 1.4$ years, height $=163.7 \pm 6.0 \mathrm{~cm}$, mass $=62.1 \pm 8.1$ $\mathrm{kg}$ ) were instrumented for biomechanical analysis. Subjects performed three sets of double-leg landings from under preferred, stiff, and soft landing conditions. A stepwise linear regression analysis revealed that ankle and knee energy absorption at impact, and knee and hip energy absorption during the stabilization phases of landing explained $75.5 \%$ of the variance in leg impedance. The primary predictor of leg impedance was knee energy absorption during the stabilization phase, independently accounting for $55 \%$ of the variance. Future validation studies applying this regression model to other groups of individuals are warranted.

Key Words: landing, lower extremity mechanics, energetic
\end{abstract}

\section{Article:}

Activities involving jumping require an applied impulse (the attenuation of the ground reaction forces [GRFs]) upon landing to effectively change the body's momentum. Repetitive changes in momentum are required in activities such as dance, basketball, and volleyball and highlight the importance of controlling mechanisms that attenuate landing impulses safely and efficiently.

Leg spring stiffness is a measure of overall lower extremity stiffness and is represented by a mass-spring model. This measure is defined as the peak GRF divided by the body's center of mass (CoM) displacement upon ground contact, and is generally used in such tasks as locomotion and hopping (McMahon \& Cheng, 1990; McMahon, Valiant, \& Frederick, 1987; Granata, Padua, \& Wilson, 2002; Padua, Arnold, Carcia, \& Granata, 2005; Farley \& Morgenroth,

\footnotetext{
${ }^{1}$ East Carolina University: Athletic Training Education Program, 251 Ward Sports Medicine Building, East Carolina University, Greenville, NC 27858

${ }^{2}$ University of North Carolina at Greensboro: Applied Neuromechanics Research Laboratory, 238 Health and Human Performance Building, University of North Carolina at Greensboro, Greensboro, NC 27402

${ }^{3}$ Westfield State College: Athletic Training Education Program, 213 Woodward Center, Westfield State College, Westfield, MA 01086.
} 
1999; Farley, Houdijk, Strien, \& Louie, 1998). Leg spring stiffness can be modulated by changing knee flexion angle and/or velocity during running (McMahon \& Cheng, 1990; McMahon et al., 1987), modifying ankle stiffness in hopping (Farley \& Morgenroth, 1999), verbal instructions influencing contact time in jumping (Arampatzis, Schade, Walsh, \& Bruggemann, 2001), and when hopping to preferred and predetermined frequencies (Granata et al., 2002; Padua et al., 2005). Based on the deceleration and acceleration phases inherent in running and hopping, these tasks involve both energy absorption and production and are thought of as energy-conservative tasks. Because of the compression and expansion of the spring- mass model during inherently energyconservative activities such as running and hopping, it may not be appropriate to use this model when examining tasks that involve only compression and energy dissipation of the spring such as in landing. When evaluating the dissipative capacity of the lower extremity to attenuate the GRFs safely and efficiently in a landing model (Lafortune, Hennig, \& Lake, 1996a; Lafortune, Lake, \& Hennig, 1996b; DeVita \& Skelly, 1992; Zhang, Bates, \& Dufek, 2000), the term leg impedance may therefore be more appropriate because the damping characteristics of the lower extremity act to decelerate the body's vertical momentum through the eccentric efforts of the ankle, knee, and hip extensor muscles, that is, energy absorption.

The functional interpretation of leg stiffness and impedance is to purportedly determine the types of biological structures that primarily contribute to attenuating the GRFs in landing (Butler, Crowell, \& McClay Davis, 2003). When leg impedance is high, small total joint displacements occur and the GRF vector is more closely aligned with the ankle, knee, and hip joints, thereby increasing the loads placed on the bony and articular cartilaginous structures through compressive mechanisms (Lafortune et al., 1996b; Butler et al., 2003). Conversely, lower levels of leg impedance involve increased joint displacements occurring over a longer landing duration, thereby allowing for more eccentric work to be done by the lower extremity musculature (Butler et al., 2003). Thus, repetitive activities involving low levels of leg impedance may place a person at risk for overuse injuries such as tendonitis. Although the stiffness or impedance characteristics of the lower extremity may be thought of as an indicator of the primary types of biological structures attenuating the GRFs, a limitation of this measure is that it does not represent specific joint contributions to attenuating the landing impulse.

Negative mechanical work or energy absorption represents the eccentric contributions of the lower extremity joint extensor muscles (DeVita \& Skelly, 1992; Zhang et al., 2000). Net internal joint powers are calculated by multiplying the net internal joint moment by the angular velocity of that joint, and energy absorption and production are calculated from the integrated power curve (Winter, 1990). Others examining energy absorption in landing have assessed only the early phase of the landing impulse, commonly referred to as the impact phase (the first $100 \mathrm{~ms}$ after impact) (Decker, Torry, Noonan, Riviere, \& Sterett, 2002; Decker, Torry, Wyland, Sterett, $\&$ Steadman, 2003). Although the impact phase encompasses the maximum GRFs, this alone may not be sufficient to assess multijoint controlling mechanisms occurring throughout the entire landing impulse because changes in energy absorption during the latter part of the landing impulse may characterize the joint strategies used to terminate the body's vertical momentum. In addition, evaluation of the entire landing impulse would yield a more complete profile of how negative mechanical work done by the extensor muscles contribute to the dissipative capacity of the lower extremity and thus leg impedance. 
While leg impedance can be regulated by muscular recruitment, muscle-specific contributions to this measure, that is, ankle plantar flexors, knee extensors, and hip extensors, cannot be assumed. The use of energy absorption as a controlling mechanism to attenuate the GRFs during both the early (first $100 \mathrm{~ms}$ after ground contact) and late (100 ms to body's maximal CoM vertical displacement) phases of landing, encompassing the entire landing impulse, may provide a useful interpretation of joint- specific contributions to leg impedance. Therefore, the purpose of this investigation was to explain the variance in leg impedance using lower extremity energy absorption from the impact and stabilization phases of landing. We hypothesized that knee energy absorption would be the primary variable explaining leg impedance. This hypothesis was based on previous research demonstrating that the knee extensors are generally primary energy absorbers in landing (Lafortune et al., 1996a), and owing to the length of the lever arms of the femur and tibia, the knee joint seems to be best situated to modulate CoM vertical displacement, as opposed to the hip and ankle, thus contributing to leg impedance.

\section{METHODS}

Design

This study followed a within-subject model, in which subjects performed drop landings under three conditions: preferred, stiff, and soft. A stepwise regression using data from all three landing conditions examined whether energy absorption at the ankle, knee, and hip for both impact and stabilization phases of landing (predictor/independent variables) could explain the variance in leg impedance (dependent variable). However, a limitation to using joint energetics as independent variables is that one cannot differentiate whether or not joint moment or joint motion are better predictors of leg impedance.

\section{Subjects}

The subject pool consisted of 20 highly trained female dancers (age $=20.3 \pm 1.4$ years, height $=$ $163.7 \pm 6.0 \mathrm{~cm}$, mass $=62.1 \pm 8.1 \mathrm{~kg}$ ) from a university dance department. These subjects were considered highly active by engaging in exercise 6.2 (1.0) days per week for a duration of 3.6 (2.0) hours per day. Any subject with a history of lower extremity injury or surgery did not qualify for inclusion in the study. Prior to participation in the study, all subjects read and signed a written informed consent form approved by the University Institutional Review Board for the Protection of Human Subjects. We selected female dancers for this study because of their high level of experience in jumping and landing styles and their ability to respond promptly to verbal instruction. Verbal instructions were used to create three leg-stiffness landing conditions (preferred, stiff, and soft); these conditions generated a spread of the data used for the regression analysis.

\section{Instrumentation}

Kinematic data for the head, thorax, pelvis, thighs, shanks, and feet were sampled at $140 \mathrm{~Hz}$ using a 3-D electromagnetic tracking system (Motion Star, Ascension Technologies, Burlington, VT) with Motion Monitor software (Innovative Sports Training, Chicago, IL). Two Type 4060nonconducting Bertec force plates (Bertec Corporation, Columbus, $\mathrm{OH}$ ) were used to acquire bilateral ground reaction forces at $1,000 \mathrm{~Hz}$.

\section{Procedures}


After obtaining informed consent, the subject's age, exercise frequency, exercise duration, height, and mass were taken and manually recorded. Subjects were then instructed in the doubleleg landing task. Subjects stood on a $60-\mathrm{cm}$ box with their toes along the front edge. Hands were placed with the thumbs anteriorly directed atop the iliac crests with fingers pointing distally. This was done to minimize the influence of arm swing on landing mechanics. Subjects were instructed to step out with one foot and drop down onto the two force plates with one foot landing on each force plate. The instructor specifically stated not to jump up or out, hop, or step down from the box. Subjects were allowed three practice trials. To standardize testing, the limb with which the subject stepped out in two of the three practice trials was denoted as the limb they would use to step out throughout testing and is referred to as the preferred limb. After practice, subjects were prepared for kinematic setup. Although the $60-\mathrm{cm}$ box does not represent a functional jumping height commonly performed by our subject sample, we felt it was necessary to standardize landing height and control for the body's vertical linear velocity and not confound the joint energetics. The $60-\mathrm{cm}$ box height has been used by others when comparing landing styles (Zhang et al., 2000) and sex (Decker et al., 2003).

Six-degree-of-freedom-position sensors (Ascension Technologies, Burlington, VT) were affixed to the subjects with double-sided tape and/or neoprene straps (thorax and skull). Sensors were affixed bilaterally on both feet (anterior mid-shaft of third metatarsal), the anterior mid-shaft of the shanks, and lateral aspects of both thighs. Three additional sensors were placed on the sacrum, spinous process of C7, and occiput of the skull. Following placement of the nine sensors, subjects were digitized. Joint centers for the ankle and knee were digitized using the centroid method (Madigan \& Pidcoe, 2003). This method estimates the center of the knee joint as the midpoint between the two points on the medial and lateral joint lines centered in the sagittal plane. The ankle joint center was estimated as the midpoint between the medial and lateral malleoli. Hip joint centers were estimated using five hip positions in flexion, abduction, extension, and on the diagonal (flexion + abduction; extension + abduction) to estimate the proximal position of the femur relative to the pelvis, which is referred to as the Leardini method (Leardini et al., 1999).

Subjects performed 10 double-leg drop landings just as they previously practiced with no further instruction other than "Drop down off the box and land as naturally as you can." Trials in which the subject hopped up or out, stepped down from the box, stutter-stepped with either foot at landing, or thumbs came off the iliac crests were considered unacceptable and subsequently deleted, and the trial was repeated. Ten acceptable trials were recorded and saved for further data reduction and analysis. The first block of 10 trials served as the preferred landing condition. Subjects were then instructed to perform 10 landings from both the stiff and soft conditions. Specific landing instructions given by the examiner for the stiff and soft conditions were as follows: stiff condition- "Land as stiff as you can. This will be a hard landing. Land so that you maximize the amount of forces you feel on your body" and soft condition- "Land as soft as you can. This should be a quiet landing. Land so that you minimize the amount of forces you feel on your body" (Prapavessis \& McNair, 1999). The stiff and soft conditions were performed in a predetermined counterbalanced order. The three conditions totaled 30 drop landings. All subjects led with their right leg (preferred limb) when initiating dropping off the box. To control for any potential right- and left- side asymmetries, we evaluated only the right leg (preferred leg) for processing and analysis. 


\section{Data Reduction}

Kinematic data were linearly interpolated to the vertical ground reaction force data at $1,000 \mathrm{~Hz}$. Force and kinematic data were low pass filtered at $60 \mathrm{~Hz}$ and $12 \mathrm{~Hz}$ respectively using a fourthorder zero- lag digital Butterworth filter. Kinematic data were interpreted using Euler angle equations (Allard, Stokes, \& Blanchi, 1995). The 3-D rotations used for the lower extremity joints followed a flexion $(Z)$, rotation $\left(Y^{\prime}\right)$, ab-/adduction $\left(X^{\prime \prime}\right)$ sequence and this same reference served as the axis configuration for the local coordinate system. The global coordinate system was defined by $(X)$ medial-lateral, $(Y)$ vertical, and $(Z)$ anterior posterior directions. All kinematic, kinetic, and energetic data were calculated from initial contact with the force plate (exceeding $10 \mathrm{~N}$ ) to when the body's CoM position reached its minimal vertical position relative to the force plates (Zhang et al., 2000). As the CoM position reaches its lowest point in landing and begins its ascent away from the force plate, the linear velocity of the body's CoM changes direction and a change of momentum has thus occurred. The ground reaction impulse during this descending time period was then subdivided into two phases: impact and stabilization. The impact phase was defined as the time from initial contact through the first $100 \mathrm{~ms}$ of the landing impulse (Decker et al., 2002; Decker et al., 2003). The stabilization phase was defined as the time from the end of the impact phase to the minimal vertical position of the body's CoM relative to the force plates.

Net internal joint moments were calculated with Motion Monitor Software using an inverse dynamics procedure based on the kinematic data, ground reaction forces, and Dempster's anthropometric data (LeVeau, 1992). By convention, net internal hip extension, knee extension, and ankle plantar flexion moments at landing were denoted to be positive (+), negative $(-)$, and positive $(+)$ respectively. Conversely, angular velocities followed a $(-),(+),(-)$ convention with respect to the hip, knee, and ankle joints. Net internal joint powers were then calculated by multiplying the joint moments by the joint angular velocities. Mechanical energy production and absorption were then calculated as the integral of the positive and negative joint power curves respectively (Winter, 1990).

Leg impedance was calculated as the peak vertical ground reaction force (vGRF) divided by the vertical displacement of the body's CoM (Farley et al., 1998; Farley \& Morgenroth, 1999). The body's CoM was estimated based on the summed mass of the nine segments digitized and represented $89.8 \%$ of total body mass (LeVeau, 1992). A limitation of this model existed as we did not have enough sensors to digitize the arms and account for $100 \%$ of the whole body's mass. However, we standardized arm swing by having the subjects place their thumbs atop their iliac crests, with fingers pointing distally. Therefore any changes in the CoM position would not be due to changes in arm position. Although landing with the arms constrained is not functional in nature and may influence whether or not a subject could land in a "natural" manner, this standardization was necessary to ensure that any changes in the body's CoM position was due to the modeled segments of interest, that is, the lower extremity and trunk positions. All joint moments, energetics, and leg impedance data were normalized to body mass. For all variables of interest, a mean from 10 trials was chosen to represent the average performance characteristics under each condition. Ten trials have been used previously in landing and hopping to estimate the average performance characteristics (Granata et al., 2002; DeVita \& Skelly, 1992). 


\section{Statistical Analyses}

Although it was not one of the purposes of this investigation to assess changes in leg impedance across condition, a repeated-measures ANOVA was performed on this variable across landing conditions to ensure that the three landing conditions (preferred, stiff, and soft) differed. A stepwise linear regression examined the variance in leg impedance explained by lower extremity energy absorption characteristics. Independent variables used for this regression analysis were hip, knee, and ankle energy absorption each during the impact phase (first $100 \mathrm{~ms}$ after impact) and stabilization phase (100 ms to body's maximal CoM vertical displacement). We utilized all three landing conditions in this analysis $(N=60)$. Stepwise linear regression examined the independent variable with the highest correlation with the dependent variable to form a regression model. Subsequent models were formed by adding the independent variable with the highest partial correlation with the dependent variable once the effect of the previous model was accounted for. This process was repeated until an additional independent variable did not produce a significant $F$ change as indicated by the ANOVA table. The repeated-measures ANOVA and stepwise linear regression analyses were performed using SPSS v.13.0 for Windows. A priori alpha levels were set at 0.05 for all analyses.

\section{RESULTS}

Repeated-measures ANOVA confirmed that leg impedance increased significantly from soft to preferred to stiff landing techniques, thus creating three different conditions, $F(2,38)=67.201, p$ $<.0001$ (Table 1 and Figure 1). Table 2 displays the means and standard deviations of the sample population for energy absorption by joint, phase of landing, and landing condition. Regression results reflect the energy absorption by joint and landing phase across all landing conditions.

The stepwise linear regression revealed that $75.5 \%$ of the total variance in leg impedance was explained by energy absorption at the knee (impact and stabilization phase), hip (stabilization phase), and ankle (impact phase), $F(4,55)=42.298, p<$

Table 1 Leg Impedance, Peak Ground Reaction Forces, Center of Mass Displacements, and Landing Impulse Times Across Landing Technique

\begin{tabular}{|c|c|c|c|c|c|c|c|c|}
\hline \multirow[b]{2}{*}{ Landing technique } & \multicolumn{2}{|c|}{$\begin{array}{l}\text { Leg impedance } \\
\left(\left[\mathrm{N} / \mathrm{m}^{\prime} \mathbf{k g}^{-1}\right)^{*}\right.\end{array}$} & \multicolumn{2}{|c|}{ GRF peak $\left(\mathbf{N} \cdot \mathrm{kg}^{-1}\right)$} & \multicolumn{2}{|c|}{$\begin{array}{l}\text { Center of mass } \\
\text { displacement (m) }\end{array}$} & \multicolumn{2}{|c|}{$\begin{array}{l}\text { Landing impulse } \\
\text { time (ms) }\end{array}$} \\
\hline & $\boldsymbol{M}$ & $S D$ & $M$ & $S D$ & $M$ & $S D$ & $M$ & $S D$ \\
\hline Preferred & 92.4 & 22.3 & 28.9 & 4.4 & .33 & .04 & 221.5 & 29.8 \\
\hline Stiff & 139.7 & 39.8 & 35.4 & 5.2 & .27 & .05 & 171.4 & 31.1 \\
\hline Soft & 68.5 & 17.3 & 24.5 & 4.0 & .37 & .05 & 259.7 & 33.2 \\
\hline
\end{tabular}

*All landing conditions are significantly different from each other, $p<.001 ; \mathrm{GRF}=$ ground reaction force. 
Table 2 Average Energy Absorption by Technique, Joint, and Phase of Landing

\begin{tabular}{|c|c|c|c|c|c|c|c|c|c|}
\hline \multirow[b]{2}{*}{ Joint } & \multirow[b]{2}{*}{ Phase } & \multicolumn{2}{|c|}{ Preferred landing } & \multicolumn{2}{|c|}{ Stiff landing } & \multicolumn{2}{|c|}{ Soft landing } & \multicolumn{2}{|c|}{ Average } \\
\hline & & $M$ & $S D$ & $M$ & $S D$ & $\boldsymbol{M}$ & $S D$ & $\boldsymbol{M}$ & $S D$ \\
\hline \multirow[t]{2}{*}{ Hip } & Impact & -0.93 & 2.02 & -0.96 & 1.97 & -0.63 & 1.60 & -0.84 & 1.85 \\
\hline & Stabilization & -0.25 & 0.40 & -0.18 & 0.47 & -0.18 & 0.22 & -0.20 & 0.37 \\
\hline \multirow[t]{2}{*}{ Knee } & Impact & -4.66 & 1.55 & -5.36 & 2.02 & -4.70 & 1.56 & -4.91 & 1.73 \\
\hline & Stabilization & -1.49 & 0.57 & -0.75 & 0.44 & -2.14 & 0.78 & -1.46 & 0.83 \\
\hline \multirow[t]{2}{*}{ Ankle } & Impact & -2.46 & 3.95 & -2.43 & 3.04 & -1.88 & 2.06 & -2.26 & 3.07 \\
\hline & Stabilization & -0.44 & 1.73 & -0.21 & 0.78 & -0.08 & 0.08 & -0.24 & 1.09 \\
\hline
\end{tabular}

Note. Energy absorption units $=\mathrm{N} \cdot \mathrm{m} \cdot \mathrm{kg}^{-1} ; N=20$ per landing condition (10-trial average per subject).

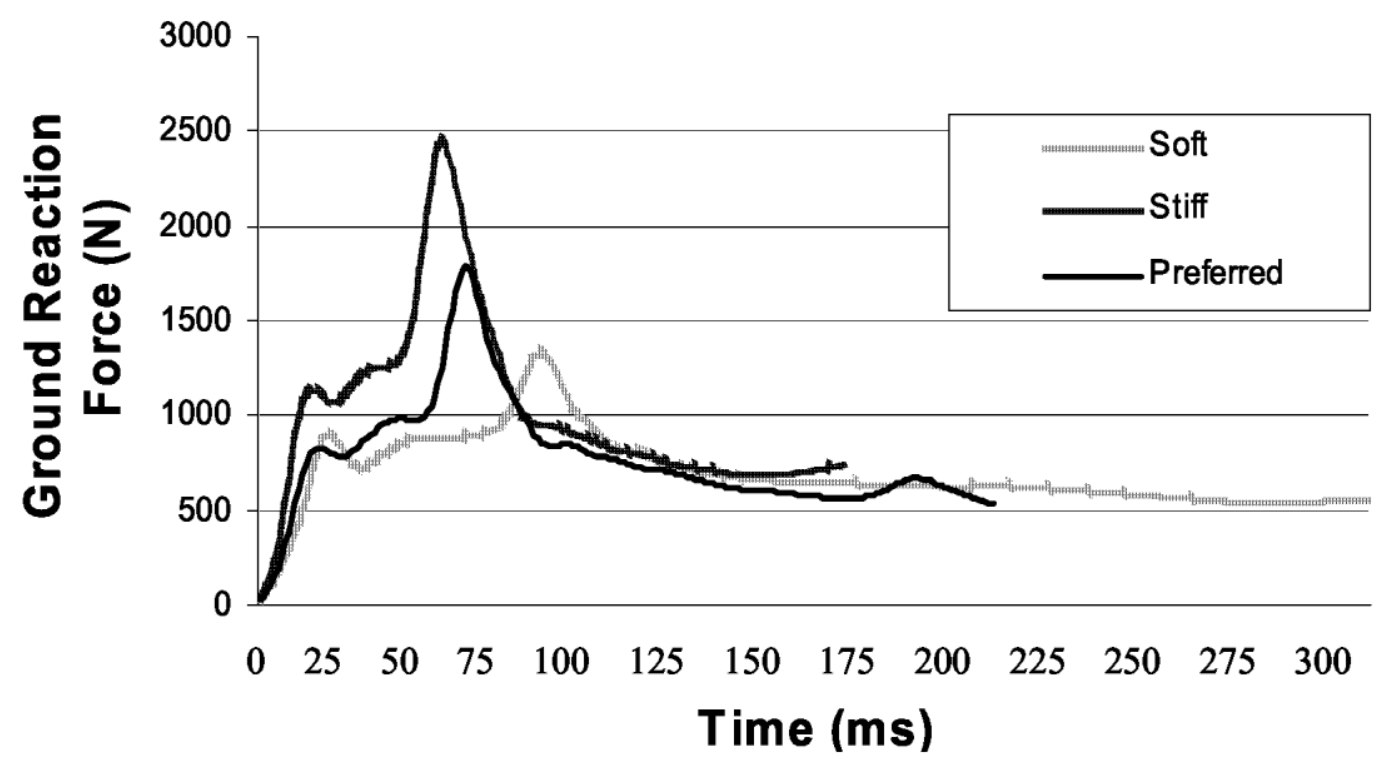

Figure 1 - Representative vertical ground reaction forces across landing technique. This figure displays one trial from each landing condition from one subject.

.0001). Knee energy absorption during the stabilization phase (KEAS) had the highest zero-order raw correlation with leg impedance and entered first, accounting for $55.1 \%$ of the variance in leg impedance $\left(r^{2}=55.1 \%, p<.001\right)$. Once the variance explained by knee energy absorption during the stabilization was accounted for, knee absorption during the impact phase (KEAI) had the next highest partial correlation, accounting for an additional $5 \%$ of the remaining variance in the data $\left(r^{2}\right.$ change $\left.=5 \%, p=.010\right)$. Hip energy absorption during the stabilization phase (HEAS) entered next $\left(r^{2}\right.$ change $\left.=8.3 \%, p<.001\right)$, followed by ankle energy absorption during the impact phase (AEAI) $\left(r^{2}\right.$ change $\left.=7.1 \%, p<.001\right)$. This four-predictor model yielded a regression equation of $113.924+35.98 *(\mathrm{KEAS})-8.2 *(\mathrm{KEAI})+60.16(\mathrm{HEAS})-4.76 *(\mathrm{AEAI})$. Table 3 provides the full model summary, and Figure 2 visually demonstrates the relationship between the actual versus predicted leg impedance values.

\section{DISCUSSION}

The primary finding of this study was that $75.5 \%$ of the variance in leg impedance was explained by a combination of energy absorption at the ankle and knee at impact, and the knee and hip 
during the stabilization phases of landing. However, the majority of leg impedance was explained by energy absorption at the knee $\left(r^{2}=60.1 \%\right.$; Table 3-Model \#2b), particularly during the stabilization phase of the landing $\left(r^{2}=55.1 \%\right.$; Table 3-Model 1a).

Table 3 Regression Model Summary

\begin{tabular}{|c|c|c|c|c|c|c|c|c|c|}
\hline \multirow[b]{2}{*}{ Model } & \multirow[b]{2}{*}{$\boldsymbol{R}$} & \multirow[b]{2}{*}{$R^{2}$} & \multirow[b]{2}{*}{ Adjusted $R^{2}$} & \multirow[b]{2}{*}{ SE of estimate } & \multicolumn{5}{|c|}{ Change statistics } \\
\hline & & & & & $R^{2}$ change & $F$ change & df1 & df2 & Sig. $F$ change \\
\hline $1 \mathrm{a}$ & 0.742 & 0.551 & 0.543 & 27.524 & 0.551 & 71.075 & 1 & 58 & 0.000 \\
\hline $2 b$ & 0.775 & 0.601 & 0.587 & 26.171 & 0.050 & 7.151 & 1 & 57 & 0.010 \\
\hline $3 c$ & 0.827 & 0.684 & 0.667 & 23.503 & 0.083 & 14.675 & 1 & 56 & 0.000 \\
\hline $4 d$ & 0.869 & 0.755 & 0.737 & 20.884 & 0.071 & 15.925 & 1 & 55 & 0.000 \\
\hline
\end{tabular}

A-Predictors: (constant), knee energy absorption stabilization.

B-Predictors: (constant), model A predictor, knee energy absorption impact.

C-Predictors: (constant), model B predictors, hip energy absorption stabilization.

D-Predictors: (constant), model C predictors, ankle energy absorption impact.

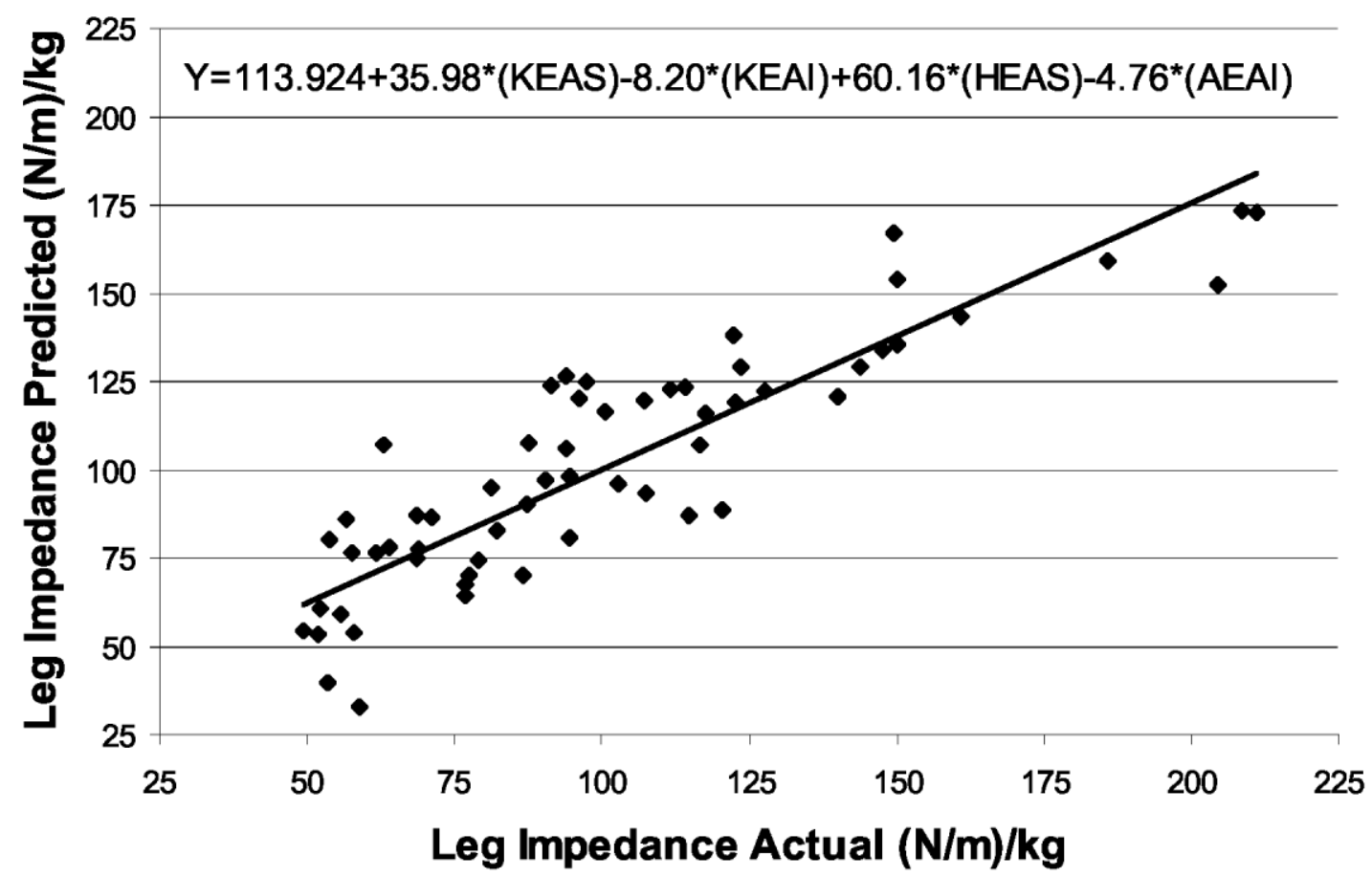

Figure 2 - Predicted versus actual leg impedance. *KEAS = knee energy absorption/stabilization phase, KEAI $=$ knee energy absorption/impact phase, HEAS = hip energy absorption/stabilization phase, AEAI = ankle energy absorption/impact phase. Full model summary for model $\mathrm{D} ;{ }_{R} 2=75.5$ (see Table 3).

Because leg impedance describes the interaction between the peak GRF and CoM displacement and the knee contributed to the largest proportion of variance explaining leg impedance, the importance of the knee joint in the attenuation of GRFs and in terminating the body's momentum is demonstrated. Based on the regression analysis, our primary hypothesis that the knee would be the primary explainer of leg impedance was supported. As energy absorption is spread over a longer landing impulse and energy absorption by the knee extensors during the stabilization 
phase increases, leg impedance decreases. The contention purported by others (Schot \& Dufek, 1993; Butler et al., 2003) that low levels of leg stiffness increases the demands on the hip, knee, and ankle extensors to attenuate the GRFs and are therefore at an increased risk for overuse injuries through repetitive mechanisms is partially supported by our regression model because low leg impedance levels are consistent with increased energy absorption at the knee and hip during the stabilization phase of landing. Because the increases in the amount of work done on the knee extensors occur during the latter stabilization phase of landing, a time when both peak knee joint flexion and maximum muscle lengthening is likely to occur, an increased risk for softtissue injuries such as a quadriceps strain or patellar tendonitis is supported. Although we did not specifically measure the muscular activity of the quadriceps directly, these comments are based primarily on the energy absorption results. Conversely, with our regression model, as energy absorption decreases during the stabilization phase and thus increases during the impact phase of the landing impulse - that is, the GRF impulse is attenuated rather quickly-leg impedance increases. So, as the landing impulse is attenuated quickly, we can speculate that there was greater co-contraction among the lower extremity muscles, that is, the quadriceps and hamstrings, which restricted the amount of joint motion and allowed the GRF vector to be more closely aligned to the lower extremity joints, thereby placing higher loads on the bony and articular cartilage. Therefore, the relationship between leg impedance and energy absorption as reported by our regression model seems to add validity to the interpretation of the types of structures involved in attenuating the GRFs at different levels of leg impedance.

The division of the landing impulse into impact and stabilization phases of landing yielded important information not only about which specific joints explained the variance in leg impedance, but also when each joint contributed during the landing impulse. For example, the knee and ankle both contributed to the model during the impact phase of landing, indicating that these joints are important in attenuating the highest GRFs upon ground contact. As the body progressed into the stabilization phase of landing, the knee and hip contributed to the model, suggesting they were the primary joints contributing to the termination of the body's CoM displacement.

Traditionally, examination of the impact phase of landing or the early parts of the landing encompassing the majority of the ground reaction impulse has been performed as the research questions have centered on the relationship between impact forces and injury (Lafortune et al., 1996a; James, Bates, \& Dufek, 2003). To date, we could locate only one study involving the assessment of the stabilization phase of a drop-landing task (McNitt-Gray, Hester, Mathiyakom, \& Munkasy, 2001). Although this study operationally defined the impact and stabilization phases differently than the current study, the purpose of including both phases was to better understand multijoint control strategies used to cause a change in the body's momentum during such tasks as drop landings and front and back saltos (McNitt-Gray et al., 2001). In the current study, it appears that the hip and knee joints were integral during the stabilization phase of landing and in terminating the body's vertical momentum. Further, as energy absorption at the knee joint during the stabilization phase of landing explained $55 \%$ of the variance in leg impedance and was the first variable entered into the model (see Table 3), we concluded that our subjects primarily utilized a knee strategy in terminating the body's momentum during the latter phase of landing. Future research assessing multi- joint postural control mechanisms during dynamic motion 
requiring control of the body's momentum should therefore consider the role of the stabilization phase of landing.

A limitation of this study is the validity of this regression model in populations other than highly trained female dancers. In fact, it is possible that our results may be restricted to the population utilized. Anecdotal observations made by the examiner throughout this study were that the subjects generally presented with an upright posture and therefore may support the small contributions of hip energy absorption demonstrated across the landing conditions. A study conducted on female basketball players across stiff and soft landing conditions demonstrated that hip energy absorption was higher (DeVita \& Skelly, 1992) than the hip energy absorption demonstrated in the current study. Because of potential differences between groups performing similar landing activities, our results cannot be generalized to other populations until further research is conducted to validate the model on these different groups. A limitation of the regression model lies in its interpretation when leg impedance was high. We observed systematically high residual errors at leg impedance levels higher than $150(\mathrm{~N} / \mathrm{m}) / \mathrm{kg}$, meaning that the regression model underpredicted leg impedance when the actual leg impedance values were greater than $150(\mathrm{~N} / \mathrm{m}) / \mathrm{kg}$. No systematic residual error bias (indicating underprediction or overproduction) was noted at lower values of leg impedance.

A limitation of the current study is that we used the average of 10 trials per landing condition per subject for the regression analysis rather than individual trial data. The within-subject variability (variability between trials) of these highly trained female dancers may have strengthened or hindered the regression analysis. However, we achieved a heterogeneous data set of leg impedance as shown in Table 1 through alterations in landing style achieved by verbal cues to land "normal," "stiff," or "soft." Nevertheless, our exclusion of individual trials in favor of using the average performances from all 10 trials is a potential limitation of our results.

The primary purpose of this investigation was to determine the relationship between leg impedance and lower extremity energy absorption in landing. Our results support that leg impedance is best explained through combined contributions of the ankle and knee during the impact phase and the knee and hip during the stabilization phase of landing. In addition, the fact that knee energy absorption during the stabilization phase of landing was the first variable entered into the regression accounting for $55.1 \%$ of the variance in leg impedance, the importance of knee energy absorption and thus the knee extensors contributing to the termination of the body's vertical momentum is highlighted. The relationship between leg impedance and energy absorption also supports that lower leg impedance characteristics signify higher demands on the active musculature during the latter phases of landing (stabilization phase) to attenuate the GRFs, whereas higher levels of impedance indicate smaller contributions by the musculature during the stabilization phase of landing.

\section{Acknowledgments}

This research investigation was funded in part by the Susan Stout Graduate Research Award at the University of North Carolina at Greensboro.

\section{REFERENCES}


Allard, P., Stokes, I.A.F., \& Blanchi, J.P. (1995). Three-dimensional analysis of human movement. Champaign, IL: Human Kinetics.

Arampatzis, A., Schade, F., Walsh, M., \& Bruggemann, G.P. (2001). Influence of leg stiffness and its effect on myodynamic jumping performance. Journal of Electromyography and Kinesiology, 11, 355-364.

Butler, R.J., Crowell, H.P., \& McClay-Davis, I. (2003). Lower extremity stiffness: Implications for performance and injury. Clinical Biomechanics, 18, 511-517.

Decker, M.J., Torry, M.R., Noonan, T.J., Riviere, A., \& Sterett, W.J. (2002). Landing adaptations after ACL reconstruction. Medicine and Science in Sports and Exercise, 34, 1408-1413.

Decker, M.J., Torry, M.R., Wyland, D.J., Sterett, W.I., \& Steadman, J.R. (2003). Gender differences in lower extremity kinematics, kinetics and energy absorption during landing. Clinical Biomechanics, 18, 662-669.

DeVita, P., \& Skelly, W.A. (1992). Effect of landing stiffness on joint kinetics and energetics in the lower extremity. Medicine and Science in Sports and Exercise, 24, 108-115.

Farley, C.T., Houdijk, H.H.P., Strien, C.V., \& Louie, M. (1998). Mechanism of leg stiffness adjustment for hopping on surfaces of different stiffnesses. Journal of Applied Physiology, 85, 1044-1055.

Farley, C.T., \& Morgenroth, D.C. (1999). Leg stiffness primarily depends on ankle stiffness during human hopping. Journal of Biomechanics, 32, 267-273.

Granata, K.P., Padua, D.A., \& Wilson, S.E. (2002). Gender differences in active musculoskeletal stiffness. Part II. Quantification of leg stiffness during functional hopping tasks. Journal of Electromyography and Kinesiology, 12, 127-135.

James, C.R., Bates, B.T., \& Dufek, J.S. (2003). Classification and comparison of biomechanical response strategies for accomodating landing impact. Journal of Applied Biomechanics, 19, 106118.

Lafortune, M.A., Hennig, E.M., \& Lake, M.J. (1996a). Dominant role of interface over knee angle for cushioning impact loading and regulating initial leg stiffness. Journal of Biomechanics, 29, 1523-1529.

Lafortune, M.A., Lake, M.J., \& Hennig, E.M. (1996b). Differential shock transmission response of the human to impact severity and lower limb posture. Journal of Biomechanics, 29, 1531-1537.

Leardini, A., Cappozzo, A., Catani, F., Toksvig-Larsen, S., Petitto, A., Sforza, V., Cassanelli, G., \& Giannini, S. (1999). Validation of a functional methods for the estimation of hip joint centre location. Journal of Biomechanics, 32, 99-103.

LeVeau, B.F. (1992). Biomechanics of Human Motion. (3rd ed.) Philadelphia, PA: W.B. Saunders.

Madigan, M.L. \& Pidcoe, P.E. (2003). Changes in landing biomechanics during a fatiguing landing activity. Journal of Electromyography and Kinesiology, 13, 491-498.

McMahon, T.A. \& Cheng, G.C. (1990). The mechanics of running: How does stiffness couple with speed? Journal of Biomechanics, 23, 65-78.

McMahon, T.A., Valiant, G., \& Frederick, E.C. (1987). Groucho running. Journal of Applied Physiology, 62, 2326-2337.

McNitt-Gray, J.L., Hester, D.M.E., Mathiyakom, W., \& Munkasy, B.A. (2001). Mechanical demand and multi- joint control during landing depend on orientation of the body segments relative to the reaction force. Journal of Biomechanics, 34, 1471-1482.

Padua, D.A., Arnold, B.L., Carcia, C.R., \& Granata, K.P. (2005). Gender differences in leg stiffness and stiffness recruitment strategy during two-legged hopping. Journal of Motor Behavior, 37, 111-125. 
Prapavessis, H. \& McNair, P.J. (1999). Effects of instruction in jumping technique and experience jumping on ground reaction forces. Journal of Orthopaedic \& Sports Physical Therapy, 29, 352356.

Schot, P.K., \& Dufek, J.S. (1993). Landing performance, part I: Kinematic, kinetic, and neuromuscular aspects. Medicine, Exercise, Nutrition, and Health, 2, 69-83.

Winter, D.A. (1990). Biomechanics and Motor Control of Human Movement. (2nd ed.) New York: Wiley-Interscience.

Zhang, S.N., Bates, B.T., \& Dufek, J.S. (2000). Contributions of lower extremity joints to energy dissipation during landings. Medicine and Science in Sports and Exercise, 32, 812-819. 